\title{
Diffuse B-Cell Lymphoma: An unusual cause of bilateral adrenal masses with adrenal insufficiency
}

\section{Dr T Crabtree ${ }^{1}$, Dr H Ali ${ }^{2}$}

1. ST4 Endocrinology \& Diabetes mellitus, University Hospitals of Derby and Burton NHS Foundation Trust

2. Consultant in Endocrinology \& Diabetes mellitus, University Hospitals of Derby and Burton NHS Foundation Trust

\section{History}

The patient was a74 year old gentleman who was initially referred to Gastroenterology with symptoms of weight loss, nausea and loss of appetite. Initial investigations were targeted towards exclusion of an underlying malignancy and identified seemingly incidental bilateral adrenal lesions. Lung nodules were also noted. Further dedicated adrenal imaging showed no interval increase in size with contrast washout $>60 \%$ and relative washout of $40 \%$ consistent with multiple bilateral adenomata

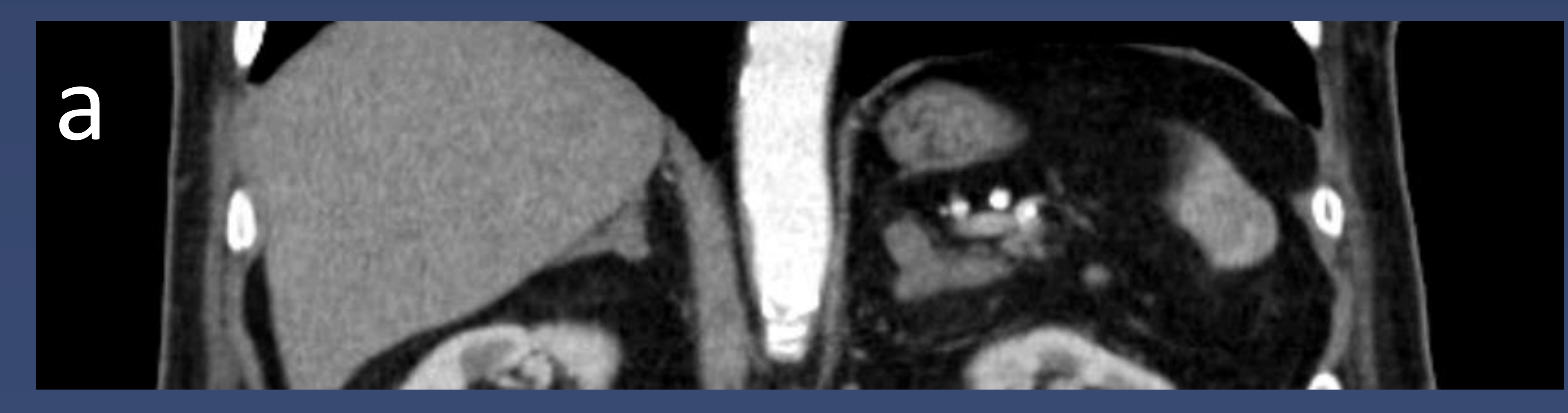

Subsequent to this he presented to A\&E following a fall and was found to be hyponatraemiac $(124 \mathrm{mmo} / \mathrm{L})$ and hyperkalaemic $(6.2 \mathrm{mmol} / \mathrm{L})$. Clinically his skin appeared tanned.

Short Synacthen was performed at this time which showed a failure of response of Cortisol ( 0 minute $75 \mathrm{nmol} / \mathrm{L} ; 30$ minute $78 \mathrm{nmol} / \mathrm{L}$ ).

Endocrine review was requested at this point and further biochemical screen was requested. Renin and Aldosterone were both normal. Urinary metanephrines were normal. He was commenced on IV Hydrocortisone and then switched to oral at a dose of 10, 5, 5 .

He was referred to the Urology MDT for discussion. The Urology MDT reviewed and suggested the CT scan findings were most consistent with hyperplasia. As the lesions were stable in size and appearance and therefore for rescan in 12 months time.

The lung nodules were also noted to be stable and were of minimal concern when discussed at Respiratory MDT.

\section{Deterioration}

The patient remained mostly well over the coming months but represented shortly after his 12 month interval scan with symptoms consistent with impending adrenal crisis. His clinical condition had changed dramatically and he was noted to be anaemic during his admission $(\mathrm{Hb} 83 \mathrm{~g} / \mathrm{dL})$ and his $\mathrm{LDH}$ was also marginally elevated (653u/L; ref $154-412 \mathrm{u} / \mathrm{L})$.

A planned laparoscopic incisional adrenal biopsy went ahead shortly following this although was complicated due to the size and position of the lesion. The patient was admitted to ITU briefly post-operatively. Histology showed diffuse large B-cell lymphoma.

\section{From the literature}

Primary adrenal lymphoma is extremely rare, although diffuse large B-cell lymphoma is the most common subtype to affect the adrenal glands. It accounts for $<1 \%$ of extranodal lymphoma. Most frequently it occurs in male patients older than the age of 70. Typically involvement is bilateral as opposed to metastatic spread which is generally unilateral. There is no recgonised correlation between tumour size and degree adrenal hypofunction though adrenal hypofunction is common.[1,2]

Prognosis is felt to be very poor and optimum chemotherapeutic regimens are yet to be established due to the paucity of evidence and infrequency of the cases encountered.[3]

From the evidence available, and our experience with this gentleman, it may be important to consider this differential in patients with new onset adrenal hypofunction and bilateral adrenal masses, especially those which grow rapidly, as presumably earlier recognition will improve outcomes.
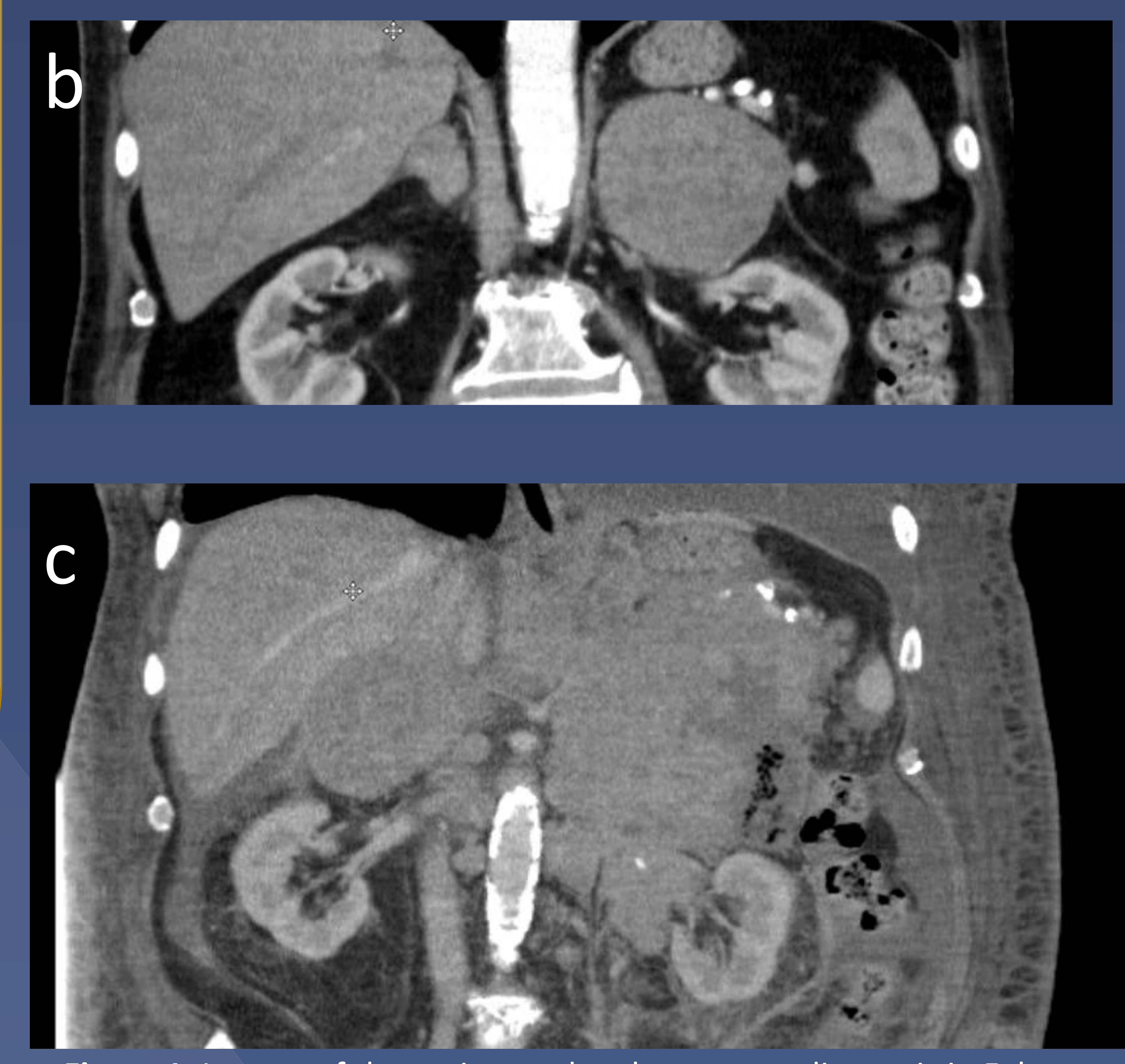

Figure 1. Images of the patients adenal masses at diagnosis in Feb 2017 (a); at interval scan in Feb 2018 (b); post-incisional biopsy (to exclude bleeding) Apr 2018 (c). Clear progression is demonstrated, observed most clearly in the left adrenal lesion.

\section{Resolution}

The patient was admitted again shortly following discharge from his biopsy. His clinical condition had deteriorated markedly and, with his biopsy results now being available, it seemed obvious he was deteriorating as a result of his advancing lymphoma. He was commenced on oral chemotherapy and a palliative approach was adopted, at this point appearing too frail for curative chemotherapeutic intervention.

Unfortunately he deteriorated rapidly and eventually passed away as a result of his condition.

\section{References}

1. Mozos A, Ye H, Chuang WY, Chu JS, Huang WT, Chen HK, Hsu YY Du MQ, Campo E, Chuang SS. Most primary adrenal lymphon as large B-cell lymphomas with non-germinal center B-cell pheno rearrangement and poor prognosis. Mod Pathol. 2009;22:1 10.1038/modpathol.2009.87

2. Kacem K, Zriba S, Lakhal RB, Bouteraa W, Aissaoui L, Amor RB, Abdennebi YB, Ali ZB, Abid HB, Meddeb B. Primary adrenal lymphoma. Turk J Hae 2014;31:188-191. doi: 10.4274/tjh.2012.0125.

3. Grigg AP, Connors JM. Primary adrenal lymphoma. Clin Lymphoma. 2003;4:154-160. doi: 10.3816/CLM.2003.n.024 